\title{
¿CÓMO SALVAR DE SÍ MISMA A LA UNIVERSIDAD? Retos y límites de la profesión académica
}

\author{
HOW TO SAVE THE UNIVERSITY FROM ITSELF?
}

Challenges and limits of the academic profession

\author{
Mariano Fernández Enguita mfenguita@edu.ucm.es \\ Universidad Complutense de Madrid. España.
}

Es difícil dejar de ver que la universidad tiene un problema. No me refiero ya a los recientes recortes presupuestarios en el sur de Europa, ni a la normativa que prepara el Ministerio, ni a las movilizaciones de estudiantes y profesores de los últimos tiempos, todo lo cual, si acaso, presenta el riesgo de hacernos creer, como tantos afirman, que simplemente estamos (hace ya decenios, según algunos, pero ahora más) ante la consabida política mercantilizadora, la eterna ofensiva neoliberal o la agresión del gobierno autonómico de turno. Algo de todo eso hay, pero el riesgo añadido es que nos impida ver los procesos más de fondo que están sacudiendo los cimientos de la vieja institución. Desde la perspectiva de mi actual y vieja universidad, la Complutense, me afectan y me preocupan las restricciones que estamos viviendo, pero lo que más me inquieta es que las respuestas más visibles vayan de los inefables llamamientos del Rectorado a la ciudadanía para que se levante en defensa de la universidad tal cual estaba a las aulas en la calle encabezadas por una de esas áreas que tienen casi tantos profesores como alumnos.

En el mundo, incluido aquí, están pasando más cosas. En parte son las que recogen Heckscher y Martín-Ríos cuando aluden a la viabilidad económica, la conexión con la sociedad o el impacto de la investigación. Yo diría que son algo más profundas y, aunque no me centraré aquí en diseccionar la crisis, hay algunos aspectos que no quiero dejar de mencionar. El primero es el coste económico. Los autores lo mencionan, pero creo que hay que ser más precisos y señalar que la fuerte expansión universitaria puede llegar a suponer costes prohibitivos, tanto si se traducen en el endeudamiento individual y familiar, lo que está sucediendo en los Estados Unidos o en Chile, donde ya cabe hablar de una burbuja (de un importante sector de población endeudado en la perspectiva de unos ingresos que la economía no les garantiza, menos aun en la crisis), como si lo hacen en gasto público, como en la mayor parte de Europa, lo que supone una notable tendencia al aumento del déficit y de la deuda del Estado. Junto al coste económico hay que considerar, además, el coste personal que supone la prolongación de la escolaridad, al menos para los no adictos, tanto más en una cultura credencialista y academicista como la nuestra, que infla la matrícula en el bachillerato y la universidad en 
detrimento de otras enseñanzas profesionales pero con el efecto paradójico de deflactar el valor absoluto de las sobreelegidas.

El segundo, y más importante, es que mucho de lo que la universidad hace puede hacerse hoy, cada vez más, por otras vías más eficaces, más eficientes y más satisfactorias. En lo que va de siglo hemos visto, por supuesto, crecer más rápidamente la enseñanza a distancia y en línea que la presencial, así como fórmulas mixtas, tanto por su menor coste como por su mayor flexibilidad. Pero en los últimos dos años están proliferando iniciativas mucho más ambiciosas que, aunque de momento son puramente tentativas y quedarán la mayoría en nada, a medio plazo van a hacer tambalearse a las universidades, al menos tal como las conocíamos. Me refiero a las plataformas de recursos abiertos lanzadas por algunas de las más prestigiosas instituciones (MITx, EdX...), a los cursos abiertos y masivos en línea ofrecidos desde viejas universidades (Miami, Stanford...) o por empresas emergentes (Coursera, Udacity...), al despliegue de una oferta docente que va desde una academia universal (Khan, P2PU, Udemy) hasta el proyecto de crear un campus IVY virtual (Minerva), a los ensayos de acreditación desde fuera de la las instituciones (digital badgets, Degreed...), etc.

No es de extrañar que la sociedad, y dentro de ella en especial, aunque por distintos motivos, las autoridades políticas y los actores sociales más dinámicos, den muestras de creciente impaciencia ante una universidad aferrada a su pasado. Si el elefante no se mueve por sí mismo, habrá que moverlo desde fuera. Entonces llegan las propuestas de someterlo a controles y directivas más exigentes, exponerlo a los incentivos y desincentivos del mercado o cualquier mezcla de ambas cosas. Llegados a este punto, nos dicen Heckscher y Martín Ríos, el remedio puede ser peor que la enfermedad. Paradójicamente, la universidad pretendería adoptar ahora el mismo modelo que las empresas, al menos las más dinámicas, ya abandonan, el de una estructuración burocrático-administrativa. La alternativa, nos dicen, es adoptar un modelo colaborativo que para la universidad se concretaría fundamentalmente en a) un propósito común, b) equipos de trabajo interdisciplinares y flexibles y c) una interacción más intensa con los sectores de fuera de la universidad interesados en esta (los stakeholders).

Heckscher y Martín Ríos parten de los modelos de regulación social que forman una triada añadiendo al par dicotómico mercados-jerarquías (o mercados-estados) los clanes (Ouchi, 1980) o las comunidades (Streeck y Schmitter, 1985), pero le añaden el desarrollo de una nueva tipología igualmente tripartita dentro de estas, desarrollada antes por uno de ellos, según la cual las comunidades podrían inclinarse hacia el mercado, tipo Gesellschaft, hacia la jerarquía, tipo Gemeinschaft, o hacia la horizontalidad, tipo colaborativo (Adler y Heckscher, 2006; Heckscher y Martín-Ríos, 2013) (todo lo cual recuerda a Hegel y Marx y su gusto por las triadas - debilidad que yo comparto-, aunque los autores se declaren fieles al marco parsoniano). La comunidad es para ellos la relación social primaria propia de las profesiones y, en general, de la economía del conocimiento (Adler, Kwon y Heckscher, 2008). La primera idea deriva explícitamente de la concepción durkheimiana de las corporaciones profesionales como contrapunto a la anomía (Durkheim, 1992) y la segunda, aun sin decirlo —al menos ahí-, se apoya en 
el concepto más reciente y en boga de las comunidades de práctica (Lave y Wenger, 1991). Ahora bien, la academia, y en particular el claustro de profesores, con su relativa autonomía corporativa, siempre ha sido una comunidad, de manera que la cuestión es evitar que se incline, sea de grado o por fuerza, del lado del mercado o de la burocracia para lograr que se centre como profesión colaborativa.

Estudiando las profesiones docentes no universitarias, concretamente los maestros de la escuela primaria (e infantil) y los profesores de secundaria, yo propuse hace algún tiempo una idea que considero parecida, la de una profesión democrática (Fernández Enguita, 2001a,b, 2009). La conciencia común y buena parte de la sociología (desde los funcionalistas hasta los radicales, por ejemplo Hughes, 1963, y Larson, 1977, respectivamente) han identificado habitualmente a las profesiones, en el sentido fuerte del término, con grupos tales como la medicina, la abogacía o la arquitectura, valorando la profesionalización de otros posibles candidatos por referencia a ellos, casi siempre en términos de desventaja, como profesiones asalariadas o incluso semi-profesiones (Etzioni, 1969). Sin embargo, Abbott (1988) señaló con contundencia que tales profesiones-modelo no eran más que un tipo dentro de éstas, las que podemos denominar liberales, pero a su lado hay que considerar otro no menos relevante, el de las profesiones organizacionales o burocráticas, tales como jueces, sacerdotes, diplomáticos, militares, policías, etc.

$\mathrm{Mi}$ argumento, al que hasta cierto punto recuerda la tipología de las comunidades de Adler y Heckscher, era y es que el profesorado no universitario es una profesión burocrática (en un sentido no necesariamente peyorativo) que, sin embargo, expresa típicamente su frustración colectiva por comparación con las profesiones liberales, en particular con la que ellos mismos consideran la profesión por excelencia, la medicina (lo que se expresa en tediosas contraposiciones entre los inexistentes consejos de pacientes y los consejos escolares, los horarios de consulta y los de tutoría, el presuntamente indiscutido diagnóstico del médico y el siempre cuestionado del maestro y, sobre todo, las diferencias en dinero, prestigio y poder entre unos y otros). Pero el modelo burocrático de profesión, probablemente válido en su momento, cuando podían servir los procedimientos homogéneos y dirigidos desde arriba (cuando se enseñaba poco a muchos, ni siquiera todos, en primaria, y mucho a pocos, en secundaria), no sirve en una sociedad diversa y cambiante en la que cada centro y cada aula son distintos de los demás en el espacio y de sí mismos en el tiempo. Por otro lado, el anhelado modelo liberal, basado en la imagen del médico (en realidad, más en la imagen que en el médico, es decir, en la imagen distorsionada e idealizada que el profesorado tiene de la profesión médica), casa mal con un servicio público, la educación, cuya calidad sobre el terreno depende cada vez menos del profesor como practicante solitario y cada vez más de su colaboración con otros profesores, con otras profesiones y con la comunidad, es decir, de la organización escolar y de su entorno institucional y social. Frente a esto proponía yo un modelo democrático de profesión (Fernández Enguita, 2001), que vendría marcado por la capacidad de trabajar en equipo dentro del centro y en red fuera de él ("democrático", pues, frente al individualismo del modelo liberal), así como abierto a la cooperación con el entorno, necesaria en un contexto de fragmentación de los servicios públicos y 
sociales y de empoderamiento de la comunidad ("democrático", así, frente al autoritarismo propio del modelo burocrático). A nadie de le escapa que la denominación tiene cierta trampa, pues ¿quién iba a oponerse a un modelo democrático?, pero lo cierto es que no encontré mejor término. Lo mismo cabe decir, por lo demás, del concepto de universidad colaborativa, pues ¿quién va a decir que no a la propuesta de colaborar? Otro paralelismo, en fin, es que tanto los Heckscher y Martín Ríos como yo terminamos, por distintos motivos, proponiendo algo que se parece mucho al modelo idealizado de las profesiones de Parsons, Wilensky y otros funcionalistas, aunque ambos con la diferencia de que ahora se trata de propuestas -más o menos fundamentadas-, no de una pretendida constatación, pues ya es un lugar común que las profesiones, todas, están muy lejos de ser lo que dicen ser.

Ahora bien, ¿se puede aspirar a la profesión "democrática", o se puede trasladar el modelo de comunidad colaborativa a la universidad? A primera vista se diría que sí, incluso que es su lugar natural. Después de todo, la comunidad colaborativa nos conduce a las redes de colaboración, las cuales nos devuelven a la universidad. Le ética hacker (Himanen, 2001) de colaboración combinada con competitividad (Coleman, 2012) procede en buena parte de la ética académica propia del intercambio y la colaboración científicos (Benkler, 2006). Pero hay dos objeciones. La primera es que, si ya lo teníamos, ¿qué necesidad hay ahora de reinventarlo o de importarlo? La objeción no es puramente retórica, pues lo que creo es que, aunque ese modelo ha sobrevivido en la actividad investigadora, no se puede decir sin más que domine la vida universitaria, pero luego volveré sobre esto. La segunda es que las empresas en las que tan exitoso parece resultar ahora, hasta el punto de haberse convertido en una nueva ortodoxia -al menos teórica - en el sector de la economía del conocimiento, siguen no dejan por ello de tener que desenvolverse en el mercado, de manera que la colaboración discurre en convivencia no sólo con la jerarquía en el interior sino con la disciplina de la competencia en el exterior.

Es más, creo que la dicotomía jerarquías-mercados puede llevarnos a desenfocar el problema. En realidad se trata de una dicotomía en el nivel micro, la disyuntiva entre que un conjunto limitado de transacciones se lleve a cabo de manera dirigida, vertical (jerarquía), o espontánea, horizontal (mercado) (Williamson, 1975). Pero hay otra, similar, en el nivel macro, estado-mercado, que se refiere al conjunto de las transacciones en la sociedad, al menos en los límites de una sociedad nacional y cada vez más en una sociedad global (Hirschman 1970; Lane, 1985). Las comunidades pueden ser una alternativa a las transacciones o los pequeños grupos de transacciones del nivel micro, pero no lo son, o no en el mismo sentido, a esos grandes espacios macro que son el mercado y el Estado. Cuando las comunidades colaborativas, las redes o los clanes se revelan como formas eficaces de coordinación lo hacen a pequeña o mediana escala (no importan cuántas veces se reproduzca en el conjunto del espacio social) dentro de esos grandes escenarios que son el mercado y el Estado, pero no lo hacen con los mismos resultados. La mejor ilustración de esto nos la puede proporcionar, por ejemplo, la diferencia entre una empresa cooperativa y una escuela o una facultad. Por muy colabora- 
tiva que quiera ser una cooperativa de trabajadores, sus relaciones con el exterior son relaciones de mercado, lo que quiere decir predominantemente competitivas con otras empresas, por más que puedan ser también de cooperación (la llamada coopetition, que podría traducirse aquí por el ambivalente término concurrencia). Una empresa cooperativa puede decidir, por ejemplo, en medio de una crisis, reducir los salarios en vez de la plantilla, lo que probablemente haría una empresa capitalista, pero no puede eludir reducir los costes salariales en su conjunto. Pero en el marco del Estado no sucede tal cosa, sino todo lo contrario. Nada impide crear o mantener imprescindibles titulaciones que no tendrán o no tienen alumnos pero permitirán ampliar o conservar la plantilla de profesores del área, ni salir a la calle gritando "Ni una escuela menos" para mantener centros económicamente ruinosos e innecesarios, ni llamar la ciudadanía a defender lo público mientras se jubila a los profesores con sesenta años y sin reducción salarial.

De hecho, las alternativas a la burocratización y la mercantilización no se ciñen a la universidad colaborativa. Por decirlo en la terminología de Heckscher y Martín Ríos, en la comunidad universitaria las alternativas a la Gemeinschaft y la Gesellschaft no se limitan a la comunidad colaborativa. Cabe también lo que valdría llamar la Körperschaft, la comunidad corporativa que trata simplemente de imponer sus propios intereses, 0 el denominador común de los mismos. Yo diría incluso que es lo que tenemos, pues ¿cómo explicar, si no, la proliferación de títulos sin alumnos, la asimilación sin más de los cuerpos de profesores de Escuela Universitaria a los de universidad, la ya absoluta endogamia localista, la no evaluación de la docencia, la tolerancia con la no investigación o la piñata de jubilaciones anticipadas, sabáticos por mera antigüedad y demás? La única explicación es la perversa combinación de financiación pública, autonomía universitaria y gestión democrática. Cualquiera de estos pilares podría servir para una buena universidad, pero no todos a la vez, pues conducen simplemente a que la corporación decida sin control cómo gastar la pólvora del Rey. Dondequiera que uno decida y otro pague habrá poco futuro.

¿Cómo salir de este círculo vicioso en el que la corporación se fagocita a sí misma, sacrificando el futuro al presente? Heckscher y Martín Ríos proponen trasladar a la academia una fórmula que ha tenido éxito en el mundo empresarial, en particular en algunas empresas que se parecen todo lo que puede llegar a parecerse una empresa a una universidad, por su volumen y por su inserción en el manejo de información y la producción de conocimiento. Pero las concreciones de la propuesta se me antojan algo débiles.

La primera es un propósito común. Si se interpreta en los términos más generales, las universidades ya lo tienen: formación, ciencia y cultura, algo que aquí argumentó elocuentemente Ortega. Pero si se trata algo más concreto, la cosa se complica, porque la Körperschaft hace sentir todo su peso. Cualquiera que haya sido testigo de un proceso electoral universitario sabe que la corporación quiere básicamente dos cosas: que la dejen como está en términos de carga de trabajo (no más horas lectivas, no cambios de asignaturas, etc.) y que la promocionen en términos de status (cada profesor quiere acceder al cuerpo siguiente). Individualmente, los profesores pueden tener toda suerte 
de proyectos, pero el proceso de decisión colectiva sólo destila propuestas de estabilización y promoción de las plantillas y nuevas construcciones. Además, los subgrupos más afectados por problemas de este tipo suelen ser con mucho los más movilizados y juegan un papel decisivo en medio de la división más ideológica o accidental del resto. El mejor ejemplo de este círculo vicioso es la actual impotencia de la mayoría de nuestros equipos rectorales, enfangados en gestionar los efectos de la propia crisis mientras las universidades de otros países buscan frenéticamente reposicionarse en el nuevo contexto global y digital.

La segunda es la interdisciplinariedad, que sería la traducción a la universidad de la apuesta de la empresa por equipos de trabajo, grupos de tareas, estructuras matriciales, comisiones ad hoc, etc. Nominalmente todo el mundo suscribiría la propuesta, aunque en la práctica las pulsiones corporativas pueden obrar en sentido contrario. De hecho, las áreas de investigación tienden a diferenciarse más dentro de la academia que fuera de ella, porque forma parte de las estrategias de ocupación de espacios institucionales y de apoyo mutuo. Y la docencia aun más, con lo que podría calificar de limpieza disciplinar, a saber, la tendencia a expulsar de las titulaciones básicas a todas las áreas que no forman parte de su núcleo duro. No obstante, cierta interdisciplinariedad resurge en los proyectos de investigación (porque es reclamada y primada por las agencias evaluadoras) y en los estudios de posgrado (porque las áreas, exhaustas en la ocupación de las titulaciones básicas, no pueden ofertarlos por sí solas, porque quieren morder en otras clientelas y porque, siendo una oferta no reglada, hay que ofrecer cosas nuevas y hay que tener en cuenta las preferencias del público).

Yo también suscribo la propuesta, por supuesto, pero me parece que hay otros dos tipos de "interdisciplinariedad" no menos necesarios. En primer lugar, entre docentes y bibliotecarios; además, con el personal de administración y servicios. En el modelo tradicional de universidad, el docente decide qué aprender, cómo cuándo, etc., lo cual incluye el recurso a los libros y otros soportes de la información, y el bibliotecario mantiene ésta organizada y ayuda en el momento de acceso final a la misma. Se puede comparar con los papeles del médico y el farmacéutico. En la era en que entramos, con la apertura de toda suerte de vías de acceso a la información y el ascenso galopante de los precios de los servicios cara a cara, lo que implica un papel mucho más activo del estudiante en la búsqueda y selección de la información, su transmisión entre pares, etc., el docente empieza a hacer de bibliotecario y el bibliotecario hace en cierto modo de docente, y creo que cualquier paso relevante hacia nuevas formas de organización del aprendizaje debe involucrar a los servicios de biblioteca y documentación. Otro tanto cabe decir de la necesidad de colaboración con y por parte de buena parte del resto de personal administrativo, por un lado por el aumento del uso, la integración y la innovación de ciertos servicios, tales como los informáticos y audiovisuales, y por otro, más prosaico, para aligerar la creciente carga administrativa del profesorado, en parte provocada por la ineficacia típica de los servicios administrativos no centralizados.

La tercera propuesta es la apertura a la intervención de otros sectores interesados, nuestros stakeholders. Una vez más sólo cabe decir que sí, pero la pregunta es cómo 
hacerlo y qué viabilidad puede tener si depende de la corporación. En la universidad se dio cabida a los estudiantes, pero no creo que se hiciera de la forma más adecuada. A falta de una visión panorámica o de una información sistemática de las que no dispongo, mi impresión que los estudiantes pesan demasiado y mal donde tal vez no hacía tanta falta, en las elecciones a rector, y demasiado poco donde más importante y útil sería en general y para ellos en particular, en los órganos responsables de la docencia, los consejos de departamento y las coordinaciones de las titulaciones. Digo lo primero porque, dadas su bajísima participación electoral y la consiguiente autonomía de algunas pequeñas asociaciones frente a su base, por un lado, y la dispersión o división del voto de los profesores, por otro, ocurre demasiado a menudo que el voto de los estudiantes sea el que decide una elección rectoral. Y digo lo segundo porque, en sentido contrario, no siempre están presentes, o no en condiciones de hacer valer sus intereses, en los departamentos que son, en última instancia, los responsables de asegurar aquello que más directamente les afecta, la calidad de la docencia.

Esto último es parte de un problema más general, manifiesto en el conjunto del sistema educativo: la resistencia del profesorado a compartir capacidad de decisión con su público. En la enseñanza primaria y secundaria, donde teóricamente los consejos escolares son los órganos máximos de gestión o de control de la gestión de los centros y tienen atribuida una larga serie de competencias, la experiencia de un cuarto de siglo es que se han visto casi enteramente vaciados de contenido por la presencia mayoritaria de profesores, su actuación corporativamente monolítica, su capacidad de manipular la normativa, su monopolio de la legitimidad sobre la base de un discurso pretendidamente experto, la asunción de hecho de competencias impropias por los claustros, etc. Las universidades, de hecho, también han tenido a menudo relaciones difíciles con sus consejos sociales, a pesar de la irrelevancia de estos.

En todo caso, las tres propuestas de Heckscher y Martín Ríos me parecen sensatas y asumibles, a reserva de lo que acabo de explicar sobre cada una de ellas. Pero ¿podemos esperar que la propia corporación las saque adelante? Mi opinión es que no por sí sola. Aquí, de nuevo, me remito a lo que en otros momentos y lugares he escrito respecto al sistema de enseñanza no universitario, aun a sabiendas de que difiere en gran medida del universitario. Con independencia de los mecanismos que cada uno de ellos adopte, que pueden ser muchos, muy variados y cambiantes, yo creo que hay tres grandes instrumentos de regulación para las grandes instituciones: el Estado, el mercado y la cultura, con esos nombres o parecidos. El Estado es la autoridad, el control, la mano de hierro; el mercado es el interés material, los (des) incentivos, la mano invisible; la cultura es la conciencia individual, la (des)aprobación del grupo, la mano intangible (tomo esta ingeniosa metáfora de Pettit, 1997; Fernández Enguita, 2007).

En la economía del conocimiento en general, y en un medio como el universitario en particular, la forma de regulación no sólo preferible (como en todas partes) sino también más eficaz es la mano intangible, una combinación de conciencia individual y cultura grupal que se adapta como un guante a condiciones de complejidad, diversidad, 
incertidumbre, cambio... Pero la mala noticia es que no surge de la nada. La mano intangible es en gran medida la interiorización individual y colectiva de lo que previamente indican la mano de hierro y la mano invisible. Kant decía, hablando precisamente de la educación, que la autonomía moral era el resultado final de pasar por las fases de la obediencia impuesta y la obediencia querida. Mutatis mutandis, los profesores somos también criaturas kantianas, que interiorizamos las normas externas, y donde dice obediencia impuesta, obediencia querida y autonomía bien podría decir la mano de hierro, la invisible y la intangible, o el Estado, el mercado y la cultura. Las estructuras y relaciones colaborativas pueden hacer maravillas en las empresas porque estas siguen sometidas a la presión exterior del mercado. De hecho ha sido en esa frontera e interfaz con el exterior donde muchas de ellas han aprendido la lección, pues, como afirma el Cluetrain Manifesto, en la era de la información "los mercados son conversaciones" (Levine et al., 2000) y los consumidores quieren voz y se convierten en prosumidores (Toffler, 1980). Las administraciones pueden también aplicar la fórmula, aunque lo hacen en menor medida y con menos éxito, porque siguen sometidas a la mano de hierro pero esta no siempre es muy eficaz. Pero ¿las universidades? En lo fundamental, el mercado es sustituido por la financiación pública, que se sienten con derecho de reclamar en nombre del bien común o del futuro, y la jerarquía se evita en el nivel macro con la autonomía y se diluye en el nivel micro con la gestión democrática.

De hecho, creo que se puede afirmar que lo que mejor funciona, sobrevive y hasta mejora (por encima de la coyuntura actual) en la universidad, que es la investigación, lo hace porque se mueve en un pseudo-mercado relativamente eficaz, el de los proyectos en convocatorias competitivas, las publicaciones, las patentes, la difusión al público, los encuentros profesionales, la presencia en los medios, etc., con la correspondiente distribución diferencial de recursos de trabajo, ingresos adicionales y prestigio personal. Sobre la base de ese mecanismo tan material, la cultura y la conciencia pueden lograr maravillas adicionales, pero en su ausencia o en su contra dudo que lo hicieran. En cambio la docencia, libre de cualquier mano de hierro o invisible (sin controles ni incentivos), escapa también por ello al alcance de la mano intangible (la cultura). A día de hoy, la universidad se revela impotente tanto ante el profesor no investigador como ante el mal-mal docente (de los que hay que decir, como de las meigas, que no creemos que existan pero, haberlos, haylos)

Entonces: ¿universidad colaborativa, profesión democrática, regeneración institucional...? ¿Hay tiempo todavía para el dolor de contrición y el propósito de enmienda? Por supuesto, pero no pensemos que la solución vendrá simple y sencillamente de un llamamiento a ponernos a tono con lo que parece estupendo: redes, pares, flujos, colaborar, compartir, etc., por bien que suene. La universidad se mueve en la ambigüedad de ser un servicio público (parte del Estado, incluso si es privada, como institución que comparte su poder delegado), que debe crear y transmitir o incorporar el conocimiento (por consiguiente, para el mercado en general y para el mercado de trabajo en particular) y que funciona como una institución dominada por una profesión, la docente, en concurrencia con algunas otras, administradores, bibliotecarios, etc. (por tanto, depen- 
diente en gran medida de la cultura de estas profesiones y de su interiorización por sus integrantes). Cualquier reforma deberá, pues, tocar las tres teclas, incluso con un toque añadido de materialismo grosero, es decir, empezando por las que preferiríamos no haber necesitado nunca.

\section{REFERENCIAS BibLIOGRÁFICAS}

Abbott, A. D. 1988. The System of Professions: An Essay on the Division of Expert Labor. Chicago: University of Chicago Press.

Benkler, Y. 2006. The Wealth of Networks: How Social Production Transforms Markets and Freedom. New Haven, Conn.: Yale University Press.

Coleman, G. 2012. Coding Freedom - The Ethics and Aesthetics of Hacking. New Jersey: Princeton UP.

Durkheim, E. 1992. Professional Ethics and Civic Morals. London: Routledge.

Etzioni, A. 1969. The Semi-professions and their Organization: Teachers, Nurses, Social Workers. NewYork: Free Press.

Fernández Enguita, M. 2001. "A la busca de un modelo profesional para la docencia: ¿liberal, burocrático o democrático?" Revista Iberoamericana de Educación, 25: 43-64.

Fernández Enguita, M. 2007. "Educar es cosa de todos: escuela, familia y comunidad." Pp. 13-32 en J. Garreta, ed., La relación familia-escuela. Lleida: Edicions de la Universitat de Lleida, [2007].

Fernández Enguita, M. 2009. "La profesión docente en España: reflexiones y sugerencias." Pp. 119-138 en M. de Puelles, ed., Profesión y vocación docente: presente y futuro. Madrid : Biblioteca Nueva : Escuela Julián Besteiro, 2009.

Heckscher, C.C., y Adler, P.S. 2006. The Firm as a Collaborative Community: Reconstructing trust in the Knowledge Economy. Oxford: Oxford University Press.

Heckscher, C., y Martin-Rios, C. 2013. "Looking back, moving forward: toward collaborative universities. Journal of Management Inquiry, 22 (1): 136-139.

Himanen, P. 2001. The Hacker Ethic, and the Spirit of the Information Age. New York: Random House.

Hirschman, A.O. 1970. Exit, Voice, and Loyalty: Responses to Decline in Firms, Organizations, and States. Cambridge, Mass: Harvard University Press.

Hughes, E. C. 1963. "Professions." Daedalus, 92 (4): 655-668.

Lane, J.-E. 1985. State and market: The politics of the public and the private. Beverly Hills: Sage Publications.

Larson, M. S. 1977. The Rise of Professionalism: A Sociological Analysis. Berkeley: University of California Press.

Lave, J., y Wenger, E. 1991. Situated Learning: Legitimate Peripheral Participation. Cambridge: Cambridge University Press. 
Levine, R. et al. 2000. The Cluetrain Manifesto: The end of Business as Usual. Cambridge, Mass: Perseus Books.

Meyer, J.W., y Rowan, B. 1977. "Institutionalized organizations: formal structure as myth and ceremony." The American Journal of Sociology, 83 (2): 340-363.

Ouchi, W.G. 1980. "Markets, bureaucracies, and clans." Administrative Science Quarterly, 25 (1): 129-41.

Pettit, P. 1999. Republicanism: a Theory of Freedom and Government. Oxford: Oxford University Press.

Streeck, W., y Schmitter, P.C. 1985. Private Interest Government: Beyond Market and State. London: Sage Publications.

Toffler, A. 1980. Third Wave. New York: Morrow.

Williamson, O.E. 1975. Markets and Hierarchies, Analysis and Antitrust Implications: A Study in the Economics of Internal Organization. New York: Free Press.

Mariano Fernández Enguita es Catedrático en la Universidad Complutense. Antes en Salamanca, donde dirigió el Departamento de Sociología, el Centro de Análisis Sociales y el Centro Cultural Hispano Japonés y creó el portal docente Demos y el de innovación Innova. Autor de una veintena de libros y tres centenares de artículos y capítulos. Actualmente investiga sobre la profesión docente y sobre aprendizaje y escuela en la era informacional. 